\title{
Erratum: "A Large and Pristine Sample of Standard Candles across the Milky Way: 100,000 Red Clump Stars with 3\% Contamination”' (2018, ApJL, 858, L7)
}

\author{
Yuan-Sen Ting (丁源森) $)^{1,2,3,4}$ (D), Keith Hawkins ${ }^{5}$, and Hans-Walter Rix ${ }^{6}$ (D) \\ Institute for Advanced Study, Princeton, NJ 08540, USA \\ ${ }^{2}$ Department of Astrophysical Sciences, Princeton University, Princeton, NJ 08544, USA \\ ${ }^{3}$ Observatories of the Carnegie Institution of Washington, 813 Santa Barbara Street, Pasadena, CA 91101, USA \\ ${ }^{4}$ Research School of Astronomy and Astrophysics, Australian National University, Cotter Road, ACT 2611, Canberra, Australia \\ ${ }^{5}$ Department of Astronomy, Columbia University, 550 West 120th Street, New York, NY 10027, USA \\ ${ }^{6}$ Max Planck Institute for Astronomy, Königstuhl 17, D-69117 Heidelberg, Germany \\ Received 2018 August 26; published 2018 September 12
}

Supporting material: machine-readable table

LAMOST provides separate individual spectra for stars that were visited more than once. We have now culled all duplicates in the original LAMOST red clump sample with the same LAMOST designation by including in the catalog only the visit with the highest signal-to-noise ratio $(\mathrm{S} / \mathrm{N})$ for each star. We have confirmed that this does not affect the APOGEE red clump catalog in the original article, as we have already taken into account the APOGEE duplicates. We have also verified that this modification does not alter the results in the Letter; if anything, excluding the lower S/N LAMOST spectra reduces the contamination rate slightly. After culling the duplicates, there are a total of 347, 727 stars in our LAMOST catalog, of which we inferred their asteroseismic parameters from the LAMOST spectra. Among these stars, we determine that 149,732 stars are red clump stars (defined through $\Delta P>250 \mathrm{~s}$ ), 51,612 of which are from LAMOST spectra with $\mathrm{S} / \mathrm{N}_{\mathrm{pix}}>75$. These 51,612 stars are identified as "RC Pristine" because their classifications are more reliable (see Figure 2 in the original Letter), and we define the other red clump (RC) stars as "RC" in the catalog. When combining with the APOGEE catalog, there are a total of 180,897 unique red clump stars with a contamination rate of $\sim 9 \%$. Most of the contamination comes from the low S/N LAMOST sample. If we restrict ourselves to LAMOST stars with $\mathrm{S} / \mathrm{N}_{\text {pix }}>75$, there are a total of 85,539 stars with a contamination of only $\sim 3 \%$. The updated Table 1 below is included in this erratum, along with its machine-readable counterpart.

Table 1

Asteroseismic Parameters Added Catalogs for LAMOST

\begin{tabular}{|c|c|c|c|c|c|c|c|c|c|}
\hline Designation & R.A. (deg) & DECL. (deg) & $\mathrm{S} / \mathrm{N}$ & $\Delta P(\mathrm{~s})$ & $\Delta \nu(\mu \mathrm{Hz})$ & Classification & $T_{\text {eff }}(\mathrm{K})^{\mathrm{a}}$ & $\log g^{\mathrm{a}}$ & $\overline{[\mathrm{Fe} / \mathrm{H}]^{\mathrm{a}}}$ \\
\hline J220430.94-011616.7 & 331.12894 & -1.27132 & 18.6 & 236.87 & 3.48 & $\cdots$ & 4940 & 2.04 & -1.36 \\
\hline $\mathrm{J} 220432.60+005112.5$ & 331.13586 & 0.85349 & 199.5 & 76.99 & 5.02 & $\cdots$ & 4725 & 2.69 & -0.45 \\
\hline $\mathrm{J} 030142.28+001548.8$ & 45.42620 & 0.26358 & 9.9 & 354.40 & 5.55 & $\mathrm{RC}^{\mathrm{b}}$ & 4901 & 2.53 & -0.57 \\
\hline $\mathrm{J} 233420.50+332151.5$ & 353.58542 & 33.36431 & 86.5 & 302.82 & 4.55 & RC_Pristine ${ }^{b}$ & 4937 & 2.47 & -0.46 \\
\hline$\cdots$ & $\cdots$ & $\cdots$ & $\cdots$ & $\cdots$ & $\cdots$ & $\cdots$ & $\cdots$ & $\cdots$ & $\cdots$ \\
\hline
\end{tabular}

Notes. Stars that have $\Delta P>250 \mathrm{~s}$ are classified as RC stars. This table is available in its entirety (with 347,727 rows) in machine-readable form.

a The stellar parameters values are adopted from LAMOST DR3.

b For LAMOST, we further distinguish RC stars with $\mathrm{S} / \mathrm{N}_{\text {pix }}>75$ to be "RC_Pristine," and RC stars with $\mathrm{S} / \mathrm{N}_{\text {pix }}<75$ to be "RC."

(This table is available in its entirety in machine-readable form.)

\section{ORCID iDs}

Yuan-Sen Ting

(丁源森) (1) https://orcid.org/0000-0001-5082-9536
Hans-Walter Rix (iD https://orcid.org/0000-0003-49969069 